\title{
A STUDY ON THE METHOD OF IMAGE PROCESSING AND FEATURE EXTRACTION FOR CUCUMBER DISEASED
}

Youwen Tian ${ }^{1, *}$, Yan $\mathrm{Niu}^{1}$, Tianlai $\mathrm{Li}^{2}$

1 Department of Information and Electric Engineering, Shenyang Agricultural University, Shenyang, Liaoning Province, P. R. China 110161

2 Department of Horticulture, Shenyang Agricultural University,Shenyang, Liaoning Province, P. R. China 110161

* Corresponding author, Address: Department of Information and Electric Engineering, Shenyang Agricultural University, Shenyang 110161, Liaoning Province, P. R. China, Tel: +86-24-88487129, Fax: +86-24-88487122, Email: youwen_tian10@163.com

Abstract: In order to improve the recognition accuracy of cucumber diseased image, a new method based on image preprocessing and extraction of texture feature had been proposed, which making full use of color information included in cucumber image. At first, vector median filter for color images was applied to remove noise, and then chromaticity moments were extracted as texture feature. Experimental results show that vector median filter for color images can remove the noise of diseased leaf with efficiency, and the adoption of chromaticity moments as texture feature of color image is simple and rapid, which had good recognition effect.

Keywords: chromaticity moments, vector median filter, cucumber disease, image processing

\section{INTRODUCTION}

The recognition of cucumber disease based on image processing has been paid attention to and studied by more and more researchers (Zhanwu Peng,2007; Xiuli Si,2006).While image processing and texture feature are the key factors of recognition in diseased cucumber(Dong Ren et al.,2007; Ran $\mathrm{Li}, 2008)$. At present most of methods by image processing and extraction of

Please use the following format when citing this chapter:

Tian, Y., Niu, Y. and Li, T., 2009, in IFIP International Federation for Information Processing, Volume 294, Computer and Computing Technologies in Agriculture II, Volume 2, eds. D. Li, Z. Chunjiang, (Boston: Springer), pp. 967-972. 
feature were based on gray images with losing abundant color information of cucumber disease image, which affected the successive recognition results. The method of image preprocessing by vector median filter and extraction of chromaticity moments as texture feature was proposed, which was proved a good basis for recognition of cucumber disease.

\section{PREPROCESSING OF CUCUMBER DISEASE IMAGE}

In processing of color image of cucumber disease, caused by collective device, environment et al., blurry edge of disease spots and spots of disease leaves always occurred. Vector median filter had been taken to enhancing image, stressing some useful information and getting rid of or weakening harmful information in this research.

The method of vector median filter for color image, takes the average of all vectors $X_{i}$ in given window to get average vector $\bar{X}$, and calculates the distance between $\mathrm{X}_{\mathrm{i}}$ (in given window) and $\bar{X}$, then takes the nearest vector as the output value for window center pixel .It supposes that a color image express by $X_{i}=\left[R_{i}, G_{i}, B_{i}\right](i=1,2, \ldots, N, N$ is pixel), for given window there are $\mathrm{s}$ vector sets, that is $\mathrm{X}=\left\{\mathrm{X}_{\mathrm{i}}\right\}(\mathrm{i}=1,2, \ldots, \mathrm{s})$, according to the definition, the algorithm of median vector for this window as follows:

(1)Calculate average vector of the window and the distance $\mathrm{S}_{\mathrm{i}}{ }^{\prime}$

$$
\begin{gathered}
\bar{r}=\sum_{i=1}^{s} r_{i} / s \quad \bar{g}=\sum_{i=1}^{s} g_{i} / s \quad \bar{b}=\sum_{i=1}^{s} b_{i} / s \\
S_{i}^{\prime}=\left\|x_{i}-\bar{x}\right\|
\end{gathered}
$$

(2)Compare the value of $S_{i}{ }^{\prime}$, select the smallest one as $S_{\text {min }}{ }^{\prime}$

(3) $X_{\min }$ corresponding to $S_{\min }{ }^{\prime}$ is the median vector value, which will be used to replace window center pixel vector.

The application of vector median filter for color image had been taken to enhancing cucumber disease image, as shown Fig.1. The experimental result indicates that vector median filter for color image can not only remove spot and noise but keep detail of edge without increasing new color. The major reason is that center pixel is replaced by min value of the nearest vector, rather than synthetic vector by $R, G, B$. 


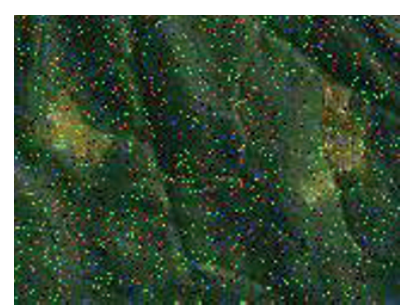

(a) Noise image

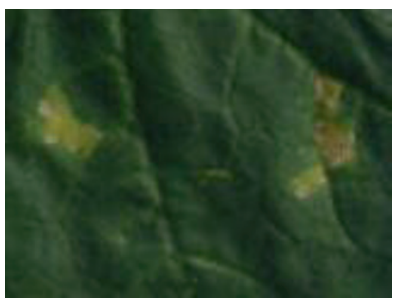

(b) Preprocessing image

Fig. 1. Experimental result of median filter for color image

\section{EXTRACTION OF CUCUMBER DISEASE FEATURE}

As the images of diseased cucumber shown, various diseased varieties have different texture and color. Hence the color texture feature of diseased cucumber was extracted for study, which became the main basis of successive recognition of cucumber disease.

\subsection{Chromaticity moments}

In this research, the selection of texture feature of color image for cucumber disease was based on chromatic concept of CIE XYZ color space. In CIE XYZ color space, every pixel has a couple chroma $(\mathrm{x}, \mathrm{y})$ derived from the XYZ color space as follows:

$$
\begin{aligned}
& x=\frac{X}{X+Y+Z} \\
& y=\frac{Y}{X+Y+Z}
\end{aligned}
$$

The chromatic diagram is, thus, a two dimensional representation of an image where each pixel produces a pair of $(\mathrm{x}, \mathrm{y})$ values. The trace of chromaticity diagram for an image is defined as follow:

$$
T(x, y)=\left\{\begin{array}{cc}
1 & \text { if }(x, y) \\
0 & \text { others }
\end{array}\right.
$$

Two-dimensional distribution (i.e., histogram) of the chromaticity diagram is defined as:

$$
\mathrm{D}(\mathrm{x}, \mathrm{y})=\mathrm{n}_{\mathrm{k}}
$$

where: $\mathrm{n}_{\mathrm{k}}$ is the producing number of $(\mathrm{x}, \mathrm{y})$ 
Now $(m, 1)$ sets of chromaticity moments are defined respectively, as:

$$
\begin{aligned}
& M_{T}(m, l)=\sum_{x=1}^{x_{s}} \sum_{y=1}^{y_{s}} x^{m} y^{l} T(x, y) \\
& M_{D}(m, l)=\sum_{x=1}^{x_{s}} \sum_{y=1}^{y_{s}} x^{m} y^{l} D(x, y)
\end{aligned}
$$

where: $\mathrm{x}_{\mathrm{s}}, \mathrm{y}_{\mathrm{s}}$ is the max value in $(\mathrm{x}, \mathrm{y})$ space.

\subsection{The analysis and comparison of different chromaticity moments}

Images with $120 * 60$ pixels can totally reflect the diseased texture feature with certain representativeness, therefore, fifty sub images with $120 * 160$ pixels had been selected as training sample from every types of disease texture image. Then the chromaticity moments sets for every texture image such as CM30, CM03, CM33, CM50, CM05, CM55 had been calculated. CM30 is expressed by $\mathrm{M}_{\mathrm{T}}(1,0), \mathrm{M}_{\mathrm{T}}(0,1), \mathrm{M}_{\mathrm{T}}(1,1)$ as feature vector, CM03 by $\mathrm{M}_{\mathrm{D}}(1,0), \mathrm{M}_{\mathrm{D}}(0,1), \mathrm{M}_{\mathrm{D}}(1,1) ; \mathrm{CM} 33$ by $\mathrm{M}_{\mathrm{D}}(1,0), \mathrm{M}_{\mathrm{D}}(0,1), \mathrm{M}_{\mathrm{D}}(1,1), \mathrm{M}_{\mathrm{T}}(1,0)$, $\mathrm{M}_{\mathrm{T}}(0,1), \mathrm{M}_{\mathrm{T}}(1,1) ; \mathrm{CM} 50$ by $\mathrm{M}_{\mathrm{T}}(1,0), \mathrm{M}_{\mathrm{T}}(0,1), \mathrm{M}_{\mathrm{T}}(1,1), \mathrm{M}_{\mathrm{T}}(2,1), \mathrm{M}_{\mathrm{T}}(1,2)$; CM05 by $M_{D}(1,0), M_{D}(0,1), M_{D}(1,1), M_{D}(2,1), M_{D}(1,2) ;$ CM55 by $M_{D}(1,0)$, $\mathrm{M}_{\mathrm{D}}(0,1), \mathrm{M}_{\mathrm{D}}(1,1), \mathrm{M}_{\mathrm{D}}(2,1), \mathrm{M}_{\mathrm{D}}(1,2), \mathrm{M}_{\mathrm{T}}(1,0), \mathrm{M}_{\mathrm{T}}(0,1), \mathrm{M}_{\mathrm{T}}(1,1), \mathrm{M}_{\mathrm{T}}(2,1)$, $\mathrm{M}_{\mathrm{T}}(1,2)$.

Then ten sub images with $120 * 160$ pixels had been selected as testing samples from every type of diseased texture image. The recognition experiment had been done by SVM with radial basic kernel function where studying parameter $\mathrm{c}=10, \zeta=0.001, \sigma=1 / 3$. The different chromaticity moments sets such as CM30, CM50, CM03, CM05, CM33, CM55 had been selected as feature vector, recognition results shown as Tabel1.

Tabell. Recognition performance of the CM method

\begin{tabular}{cccc}
\hline Types & The numbers of SVM & Correct rate of recognition & Running time $(\mathrm{ms})$ \\
\hline CM30 & 93 & $70 \%$ & 47 \\
CM50 & 85 & $70 \%$ & 47 \\
CM03 & 83 & $80 \%$ & 47 \\
CM05 & 85 & $83.3 \%$ & 62 \\
CM33 & 68 & $86.7 \%$ & 47 \\
CM55 & 75 & $90 \%$ & 47 \\
\hline
\end{tabular}

It can be concluded that when only selecting $\mathrm{M}_{\mathrm{T}}$ as the feature vector, recognition results had bad performance, and the correct rate of recognition was no more than $70 \%$. When adding $\mathrm{M}_{\mathrm{D}}$ as feature vector, the correct rate of recognition on testing sample improved. For $\mathrm{M}_{\mathrm{T}}$ only expressed if a chromatic value was existing or not, texture image with same $(\mathrm{m}, 1)$ order 
may had different textures, while $\mathrm{M}_{\mathrm{D}}$ included distribution characteristic of chromaticity, and similar color texture images had same $\mathrm{M}_{\mathrm{D}}$. There are great differences between different color texture images in $\mathrm{M}_{\mathrm{D}}$. The correct rate of recognition of using $\mathrm{M}_{\mathrm{T}}$ or $\mathrm{M}_{\mathrm{D}}$ alone was lower than the two sets of moments combined. More over, CM55 had easy calculation and good recognition performance. So that selected chromaticity moments sets CM55 as feature vector was ideal.

\subsection{Analysis of chromaticity moments invariance}

Rotation, translation, enlargement, reduction had been done to cucumber texture images shown as Fig.2. Ten sub images with $120 * 160$ pixels were selected as testing sample from every transformed images, while training sample were same to previous experiment. The recognition experiment had been done by SVM with radial basic kernel function, where $\mathrm{c}=10, \zeta=0.001, \sigma=1 / 3$. The recognition results were shown as Table2.

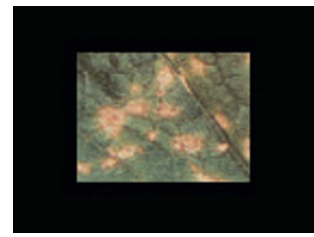

(a) Original image

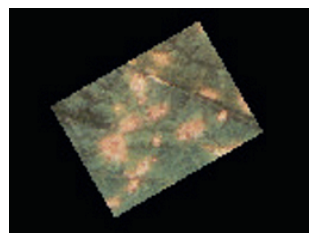

(b) Rotation

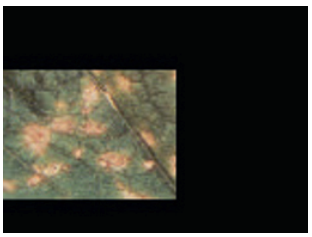

(c) Translation

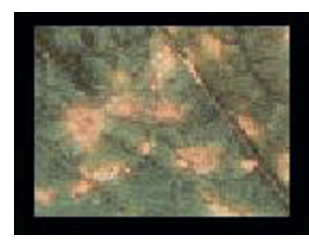

(d)Enlargement

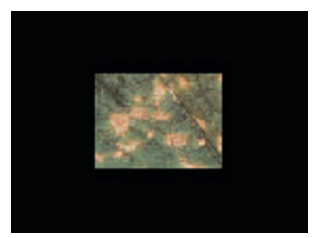

(e) Reduction

Fig.2. Transform and scaling image of cucumber disease

The data of column 2 to 4 of Tabel 2 was recognition result. The right of "/" stands for the test sample numbers of every kind of disease, and the left side stands for the numbers of being recognized correctly.

Seen from Tabel2, the recognition results changed little when previous deformation occurring in images. $\mathrm{M}_{\mathrm{T}}, \mathrm{M}_{\mathrm{D}}$ were only related to the color and color distribution of images, which having no reflected to the position of color. Thus, color and color distribution of images had no change when rotation, translation, enlargement occurring, that is, the recognition results had no change; while reduction occurring, color distribution had few differences which resulted in little change in recognition result. 
Tabel2.Recognition about invariance of chromaticity moments

\begin{tabular}{cccc}
\hline \multirow{2}{*}{ Transformed types } & \multicolumn{3}{c}{ Disease types } \\
\cline { 2 - 4 } & Power mildew & Downy mildew & Black spot \\
\hline rotation & $10 / 10$ & $8 / 10$ & $9 / 10$ \\
translation & $10 / 10$ & $8 / 10$ & $9 / 10$ \\
enlargement & $10 / 10$ & $8 / 10$ & $9 / 10$ \\
reduction & $9 / 10$ & $7 / 10$ & $9 / 10$ \\
\hline
\end{tabular}

\section{CONCLUSION}

This paper presents a method for enhancing the diseased plant image by vector median filter, extracting the texture feature vector of color image by chromaticity moments in color space CIE XYZ. The analysis of diseased cucumber experiment shows that vector median filter for color image have fast calculation, and the effect of filter is ideal. Extraction of the texture feature vector of color image by chromaticity moments is fast, which provided important parameters to successive recognition on cucumber disease.

\section{ACKNOWLEDGEMENTS}

The work is supported by the Natural Science Foundation of Liaoning Province (Item Number:20052125) and key Technologies R\&D Programme of the Educational Department of Liaoning Province(Item Number: 2005367).

\section{REFERENCES}

Dong Ren, Haiye Yu, Xiaojun Qiao. Research on Cucumber Disease Recognition in Greenhouse Based on Support Vector Machine, Journal of Agricultural Mechanization Research, 2007, 3:25-27(in Chinese)

Ran Li. Preprocessing of Leaf Image Based on Mathematical Morphology, Agriculture Network Information, 2008, 1:43-45(in Chinese)

Xiuli Si. A Study of Image Processing and Identifying Technology on Cucumber Disease. Jilin Agricultural University Master's degree paper, 2006, 1-4 (in Chinese)

Zhanwu Peng. Research on Cucumber Disease Identification Based on Image Processing and Pattern Recognition Technology. Jilin Agricultural University Master's degree paper, 2007, 1-6 (in Chinese) 